\title{
Adjusting the incidence increase when screening for statistical lead time will always give estimates of overdiagnosis close to zero
}

\author{
Per-Henrik Zahl
}

See related research by Duffy and Parmar, http://breast-cancer-research.com/content/15/3/R41

By analysing a theoretical cohort, Duffy and Parmar [1] find $0 \%$ overdiagnosis when screening for breast cancer in the UK. The fundamental assumption in their calculation is that the average lead time is 40 months, and this estimate relies on a method proposed by Walter and Day [2] for calculating lead time.

Clinical lead time and statistical lead time are two fundamentally different concepts. Clinical lead time is the time diagnosis of a clinical relevant disease is moved by screening [3]. Statistical lead time is the time diagnosis is being moved by screening when all tumours grow and there are no competing causes of death [2]. It is a theoretical concept including both clinical cancers that are detected earlier and overdiagnosed tumours. Statistical lead time for breast cancer varies between 2 and 7 years (depending on the level of overdiagnosis), while the clinical lead time is about 1 year [3]. If you adjust the incidence increase when screening for statistical lead time, it will always give estimates of overdiagnosis close to zero because you subtract all clinical cancers that are diagnosed earlier as well as all overdiagnosed cancers from the incidence increase during screening [3].

In my opinion, the authors incorrectly assume that lead time is 40 months and postulate an $86 \%$ reduction in breast cancer rate between the first two screening rounds [1]. The literature on estimates of breast cancer rates between two screening rounds (so-called interval cancer rate), which reflects the clinical lead time, reports that the decline was $48 \%$ based on a small data set [4] and close to $0 \%$ in a large British data set [5]. Tables two and three in [1] do not represent real breast cancer data. It is a constructed data set that, by definition, yields $0 \%$ overdiagnosis.

Ten years after screening they postulate a $7 \%$ reduction in incidence. However, after 20 years with overdiagnosis and overtreatment, a small reduction in breast cancer incidence is expected simply because many healthy women have lost their breast and are using antioestrogens, both of which reduce the numbers of women at risk of breast cancer. The reduction is not the effect of early diagnosis but of overtreatment or, at best, what could be called prophylactic treatment.

Finally in Table four in [1], the authors argue that the level of overdiagnosis adjusted for statistical lead time varies with length of screening as well as the length of follow-up after last screening (because the denominator varies). This is actually an argument for not using this method. Overdiagnosis adjusted for clinical lead time is insensitive to these effects [3].

\section{Authors' reply \\ Stephen W Duffy}

Dr Zahl claims that we estimate $0 \%$ overdiagnosis in breast cancer screening. In fact, our paper does not estimate overdiagnosis at all. It calculates what we would expect to happen to breast cancer incidence from lead time alone and demonstrates that if we were to estimate

Correspondence: per-henrik.zahl@fhi.no

Norwegian Institute of Public Health, PO Box 4404 Nydalen, N-0403 Oslo, Norway overdiagnosis from incidence alone, we would need long follow-up to deal with this lead time effect.

Dr Zahl's main point is that what he calls 'statistical' estimates of lead time are exaggerated, leading to underestimation of overdiagnosis, due to inclusion of overdiagnosed cancers in the estimation. However, we have observed estimates of lead time similar to the value used in our paper that do not use overdiagnosed cancers in the 
estimation, either by explicitly dividing the screen-detected cancers in the estimation [6,7] or by use of interval cancer rates alone [8], which are not screen-detected and therefore cannot include overdiagnosed cases. A more careful reading of the literature is suggested. It is also worth noting that others estimate rather longer lead times than that assumed in our paper [9].

Our estimated lead time is based on observation in randomised trials of screening. Dr Zahl's preferred method of estimation, which he refers to as 'clinical' lead time, seems to have a large number of statistical manipulations and assumptions that we find difficult to verify [3].

Our postulated population was one with $100 \%$ attendance at every screen in a 2-yearly programme, as we noted in our paper, and so of course has different numbers to those in the 3-yearly UK programme with 70 to $75 \%$ attendance. Our conclusion still stands: to estimate screening-induced overdiagnosis from incidence rates alone requires long follow-up to be reliable, and overdiagnosis has been exaggerated in the past due to insufficient duration of observation.

\section{Competing interests}

The authors declare that they have no competing interests.

Published: 04 Jun 2014

\section{References}

1. Duffy SW, Parmar D: Overdiagnosis in breast cancer: the importance of length of observation period and lead-time. Breast Cancer Res 2013, 15:R41.

2. Walter SD, Day NE: Simplified models of screening for chronic disease: estimation procedures from mass screening programmes. Biometrics 1984, 40:1-14

3. Zahl P-H, Jørgensen KJ, Gøtzsche PC: Overestimated lead-time in cancer screening has led to substantial under-estimating of overdiagnosis. Br J Cancer 2013, 109:2014-2019.

4. Woodman CB, Threlfall AG, Boggis CR, Prior P: Is the three year breast screening interval too long? Occurrence of interval cancers in NHS breast screening programme's north western region. BMJ 1995, 310:224-226.

5. Vaidya JS: Women undergoing screening mammography experience a higher incidence of invasive breast cancer, without a corresponding reduction in symptomatic breast cancer. BMJ 2009, http://www.bmj.com/ rapid-response/2011/11/02/women-undergoing-screening-mammographyexperience-higher-incidence-invasiv.

6. Olsen AH, Agbaje OF, Myles JP, Lynge E, Duffy SW: Overdiagnosis, sojourn time and sensitivity in the Copenhagen Mammography Screening Programme. Breast J 2006, 12:338-342.

7. Duffy SW, Agbaje O, Tabar L, Vitak B, Bjurstam N, Björneld L, Myles JP, Warwick J: Estimates of overdiagnosis from two trials of mammographic screening for breast cancer. Breast Cancer Res 2005, 7:258-265.

8. Paci E, Duffy SW: Modelling the analysis of breast cancer screening programmes: sensitivity, lead time and predictive value in the Florence District Programme (1975-1986). Int J Epidemio/ 1991, 20:852-858.

9. Weedon-Fekjaer $\mathrm{H}$, Lindqvist BH, Vatten $L$, Aalen OO, Tretli S: Estimating mean sojourn time and screening sensitivity using questionnaire data on time since previous screening. J Med Screen 2008, 15:83-90.

\section{$10.1186 / \mathrm{bcr} 3671$}

Cite this article as: Zahl: Adjusting the incidence increase when screening for statistical lead time will always give estimates of overdiagnosis close to zero. Breast Cancer Research 2014, 16:404 